\title{
An Absorption Correction Formula for X-Ray Fluorescence Analysis Using Compton Scattering
}

\author{
Takuji Uı ${ }^{\dagger}$, Masanao Kato and Eiichi AsAdA \\ Toyohashi University of Technology, Toyohashi 440, Japan
}

\begin{abstract}
A correction method for X-ray fluorescence analysis of the heaviest element in a light element matrix was developed on the basis of the linear relationship between the Compton scattering intensity and the reciprocal of mass absorption coefficient. Using the equation obtained here, the heaviest element in the sample was determined over a large concentration range by measuring only the Compton scattering intensity of the primary X-rays and the fluorescent $\mathrm{X}$-ray intensity for the analyte element. In this study, we employed copper in copper compounds as analyte element; the obtained copper concentrations agreed with the values calculated from chemical composition within $5 \%$ relative errors.
\end{abstract}

Keywords X-Ray fluorescence, absorption correction, light element matrix, Compton scattering, copper determination

In the X-ray fluorescence analysis of heavy elements in a light element matrix, a method employing Compton scattering X-rays as an internal standard ${ }^{1,2}$ can be used in addition to conventional calibration procedures. This method is based on the following theoretical assumptions:

1) the intensity of Compton scattering line is linearly related to the reciprocals of mass absorption coefficients for the analyte line wavelength as well as the Compton scattering wavelength;

2) in the X-ray fluorescence analysis of heavy elements in a light element matrix, only the absorption effect needs to be taken into account.

Assumption 1) is valid only when there is no major absorption edge between the Compton scattering line and the fluorescent $\mathrm{X}$-rays used as an analyte line. However, this major absorption edge cannot be neglected in the general case as shown in Fig. 1, when an $\mathrm{X}$-ray source of short wavelength such as $\mathrm{Rh}_{\alpha}$ is used. In a previous paper $^{3}$, we have described a convenient method for determination of the heaviest element in a sample under the conditions where a major absorption edge is present between the Compton scattering line and the fluorescent $X$-rays, and we have reported the application data on the analysis of iron in iron compounds.

Here a brief outline of the principles is given and results of application to the determination of copper in copper compounds are described.

The equation for absorption correction was obtained on the basis of the following three relations:

$\dagger$ Present address: Numazu College of Technology, Numazu 410, Japan
1) the difference of the mass absorption coefficients of the sample between the longer and the shorter wave-

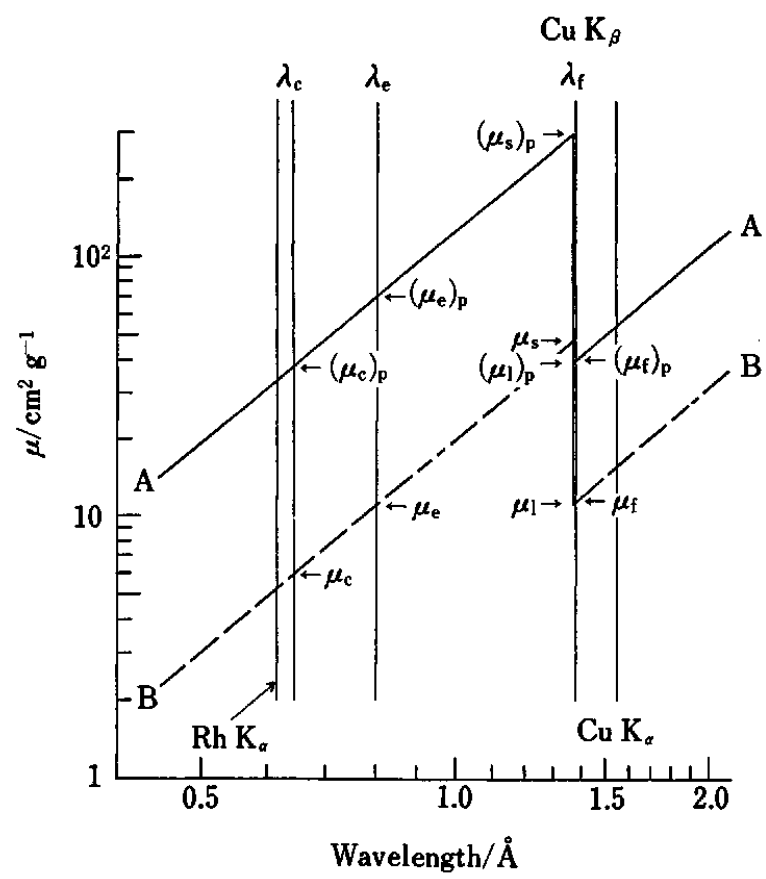

Fig. 1 Notation of wavelengths and mass absorption coefficients: $\lambda_{c}$, Rh K $K_{\alpha}$ Compton wavelength; $\lambda_{e}$, equivalent wavelength; $\mu_{\mathrm{c}}$, mass absorption coefficient for Compton scattering; $\mu_{\mathrm{e}}$, that for equivalent wavelength; $\mu_{\mathrm{s}}$, that for short wavelength side of absorption edge; $\mu_{1}$, that for long wavelength side of absorption edge; $\mu_{\mathrm{f}}$, that for analyte line. Curve A, $(\mu)_{\mathrm{p}}$ 's for pure copper; curve B, $\mu$ 's for copper gluconate. 
length sides of the absorption edge is proportional to the concentration of the analyte element whose absorption edge exists there;

2) the ratio of the mass absorption coefficients of the sample to those of the element concerned is almost constant on each side of the absorption edge (Hower's relation ${ }^{4}$;

3) an equivalent wavelength is used in polychromatic excitation. ${ }^{5}$

In order to use this equation, only the measurements of the intensities of the Compton scattering line and of the fluorescent X-rays for the analyte are required. In this study, we employed copper as the analyte element, and examined the validity of the equation.

\section{Theory}

We assumed that the heaviest element contained in the sample is the analyte element (see Fig. 1). When the mass absorption coefficient of the sample at the short wavelength side of the absorption edge is denoted by $\mu_{\mathrm{s}}$, and that at long wavelength side by $\mu_{1}$, respectively, the difference between them can be represented by the following equation:

$$
\mu_{\mathrm{s}}-\mu_{1}=\left[\Sigma\left(\mu_{\mathrm{s}}\right)_{\mathrm{i}} C_{\mathrm{i}}+\left(\mu_{\mathrm{s}}\right)_{\mathrm{p}} C\right]-\left[\Sigma\left(\mu_{1}\right)_{\mathrm{i}} C_{\mathrm{i}}+\left(\mu_{1}\right)_{\mathrm{p}} C\right],
$$

where $C$ is the weight fraction of the analyte element contained in the sample. Subscript $p$ represents the pure analyte element and $i$ the matrix element, respectively. Since the mass absorption coefficients of the matrix element will not vary at the absorption edge,

$$
\Sigma\left(\mu_{\mathrm{s}}\right)_{\mathrm{i}} C_{\mathrm{i}} \simeq \Sigma\left(\mu_{\mathrm{l}}\right)_{\mathrm{i}} C_{\mathrm{i}}
$$

Then Eq. (1) can be rewritten as

$$
\mu_{\mathrm{s}}-\mu_{1}=\left[\left(\mu_{\mathrm{s}}\right)_{\mathrm{p}}-\left(\mu_{1}\right)_{\mathrm{p}}\right] C .
$$

Applying Hower's relation to the mass absorption coefficients for wavelength shorter than that of the absorption edge, we have

$$
\mu_{\mathrm{s}} /\left(\mu_{\mathrm{s}}\right)_{\mathrm{p}}=\mu_{\mathrm{c}} /\left(\mu_{\mathrm{c}}\right)_{\mathrm{p}}
$$

where $\mu_{\mathrm{c}}$ is the mass absorption coefficient of each specimen at the Compton scattering wavelength. Rewriting Eq. (4) and using the proportionality constant $k_{\mathrm{s}}$, we have

$$
\mu_{\mathrm{s}}=k_{\mathrm{s}} \mu_{\mathrm{c}}, \quad \text { where } \quad k_{\mathrm{s}}=\left(\mu_{\mathrm{s}}\right)_{\mathrm{p}} /\left(\mu_{\mathrm{c}}\right)_{\mathrm{p}}
$$

which shows that the mass absorption coefficient $\mu_{\mathrm{s}}$ of the sample is represented by $\mu_{\mathrm{c}}$ and $k_{\mathrm{s}}$ obtained from a pure substance of the analyte element. In this equation, $\mu_{\mathrm{c}}$ can be obtained from the intensity of the Compton scattering line.

In the case of polychromatic excitation and a sufficiently thick sample, the following relation between the mass absorption coefficient $\mu_{\mathrm{e}}$ for the equivalent wavelength $\lambda_{\mathrm{c}}$ and $\mu_{\mathrm{f}}$ for the fluorescent $\mathrm{X}$-rays $\lambda_{\mathrm{f}}$ can be derived from the general formula for primary fluorescence

$$
\frac{\mu_{\mathrm{e}}+\left(\sin \phi_{1} / \sin \phi_{2}\right) \mu_{\mathrm{f}}}{\left(\mu_{\mathrm{e}}\right)_{\mathrm{p}}+\left(\sin \phi_{1} / \sin \phi_{2}\right)\left(\mu_{\mathrm{f}}\right)_{\mathrm{p}}}=\frac{(I)_{\mathrm{p}}}{I} C .
$$

Here $I$ is the intensity of fluorescent X-rays of the analyte in the sample, $(I)_{\mathrm{p}}$ that of the pure element, $\phi_{1}$ the incident angle of primary X-rays, and $\phi_{2}$ the emergence angle of fluorescent X-rays. To simplify the expression of Eq. (6) using a geometrical factor $A$ $\left(=\sin \phi_{1} / \sin \phi_{2}\right), \mu^{*}\left(=\mu_{\mathrm{e}}+A \mu_{\mathrm{f}}\right)$ and $R_{\mathrm{f}}\left(=I /\left(D_{\mathrm{p}}\right)\right.$, we have

$$
\frac{\mu_{\mathrm{e}}+A \mu_{\mathrm{f}}}{\left(\mu_{\mathrm{e}}\right)_{\mathrm{p}}+A\left(\mu_{\mathrm{f}}\right)_{\mathrm{p}}}=\frac{\mu^{*}}{\left(\mu^{*}\right)_{\mathrm{p}}}=\frac{C}{R_{\mathrm{f}}} \text {. }
$$

Applying Hower's relation to $\mu_{\mathrm{e}}$ and $\mu_{\mathrm{c}}$, we have

$$
\mu_{\mathrm{e}} / \mu_{\mathrm{c}}=\left(\mu_{\mathrm{e}}\right)_{\mathrm{p}} /\left(\mu_{\mathrm{c}}\right)_{\mathrm{p}}=k_{\mathrm{e}}
$$

and also for $\mu_{\mathrm{f}}$ and $\mu_{1}$

$$
\mu_{\mathrm{f}} / \mu_{\mathrm{l}}=\left(\mu_{\mathrm{f}}\right)_{\mathrm{p}} /\left(\mu_{\mathrm{l}}\right)_{\mathrm{p}}=k_{\mathrm{f}} .
$$

If we combine Eqs. (3), (5), (7), (8) and (9), the weight fraction can be represented as

$$
C=\frac{\left(k_{\mathrm{e}}+A k_{\mathrm{s}} k_{\mathrm{f}}\right) \mu_{\mathrm{c}}}{\left(\mu^{*}\right)_{\mathrm{p}} / R_{\mathrm{f}}+A k_{\mathrm{f}}\left[\left(\mu_{\mathrm{s}}\right)_{\mathrm{p}}-\left(\mu_{1}\right)_{\mathrm{p}}\right]} .
$$

In this equation, $\mu_{\mathrm{c}}$ and $R_{\mathrm{f}}$ are values ralated to an unknown sample, and all other factors except for $A$ and $\lambda_{e}$ are values obtained from the mass absorption coefficients of analyte element. In these equations, factor $\boldsymbol{A}$ is determined by the optical geometry, and $\lambda_{\mathrm{e}}$ is estimated on the basis of some representative samples. We can use the equations by measuring the intensities of Compton scattering and fluorescent $\mathrm{X}$-rays of pure analyte element and samples.

\section{Experimental, Results and Discussion}

The testing conditions are listed in Table 1. Samples listed in Table 2 were ground in an agate mortar, and molded in an aluminum ring of $3 \mathrm{~cm}$ in diameter under a pressure of $1 \times 10^{3} \mathrm{~kg} / \mathrm{cm}^{2}$.

\section{Determination of geometrical factor $A$}

In order to obtain $A$ in Eq. (7), the wavelengths of Compton scattering of $\mathrm{Rh} \mathrm{K}_{\alpha}$ were measured using copper gluconate. The Compton wavelength was $0.643 \AA$, and the scattering angle $\phi\left(=\phi_{1}+\phi_{2}\right)$ was obtained as $97.9^{\circ}$ from the following equation

$$
\lambda_{\mathrm{c}}-\lambda_{\mathrm{R}}=0.02426(1-\cos \phi) \text {, }
$$


Table 1 The testing conditions

\begin{tabular}{ll}
\hline Spectrometer & Phillips PW 1540 \\
Applied potential & $50 \mathrm{kV}$ \\
Tube current & $21 \mathrm{~mA}$ \\
Target & $\mathrm{Rh}$ \\
Analyte line & $\mathrm{Cu} \mathrm{K}$ \\
Analyzing crystal & $\mathrm{LiF}$ \\
Detector & scintillation counter \\
Counting time & $100 \mathrm{~s}$ for $\mathrm{Rh} \mathrm{K}_{\alpha}$ Compton scattering \\
& $100 \mathrm{~s}$ for Cu K \\
& $10 \mathrm{~s}$ for background at $41.43^{\circ} 2 \theta$ \\
Copper compounds & reagent grade \\
\hline
\end{tabular}

Table 2 Copper compounds used in this work

\begin{tabular}{rll}
\hline No. & \multicolumn{1}{c}{ Reagent } & \multicolumn{1}{c}{ Chemical formula } \\
\hline 1 & Copper(l) oxide & $\mathrm{Cu}_{2} \mathrm{O}$ \\
2 & Copper(II) oxide & $\mathrm{CuO}$ \\
3 & Copper(II) sulfide & $\mathrm{CuS}$ \\
4 & Copper(I) chloride & $\mathrm{CuCl}$ \\
5 & Copper(II) chloride & $\mathrm{CuCl}_{2} \cdot 2 \mathrm{H}_{2} \mathrm{O}$ \\
6 & Copper(II) citrate & $\mathrm{Cu}_{2} \mathrm{C}_{6} \mathrm{H}_{4} \cdot 2.5 \mathrm{H}_{2} \mathrm{O}$ \\
7 & Copper(II) acetate, cryst. & $\mathrm{Cu}_{2}\left(\mathrm{CH}_{3} \mathrm{COO}\right)_{2} \cdot \mathrm{H}_{2} \mathrm{O}$ \\
8 & Copper(II) sulfate, cryst. & $\mathrm{CuSO}_{4} \cdot 5 \mathrm{H}_{2} \mathrm{O}$ \\
9 & Copper(II) potassium chloride & $\mathrm{CuCl}_{2} \cdot 2 \mathrm{KCl}_{2} \cdot 2 \mathrm{H}_{2} \mathrm{O}$ \\
10 & Copper(II) gluconate & $\left(\mathrm{C}_{6} \mathrm{H}_{11} \mathrm{O}_{7}\right)_{2} \mathrm{Cu}$ \\
11 & Copper sheet & $\mathrm{Cu}$ \\
\hline
\end{tabular}

where $\lambda_{\mathrm{c}}$ is the wavelength of the Compton scattering and $\lambda_{\mathrm{R}}$ that of the coherent scattering, $0.613 \AA$.

The angle $\phi_{1}$ and $\phi_{2}$ were estimated on the basis of instrumental design as follows: The angle $2 \theta$ was $73.0^{\circ}$, when the analyzing crystal was kept parallel to the surface of sample holder. We obtained $\phi_{2}$ as $36.5^{\circ}$ and $\phi_{1}$ as $61.4^{\circ}$. Then coefficient $A$ becomes 1.476.

\section{Estimation of equivalent wavelength}

Equation (7) shows that $\mu^{*} /\left(\mu^{*}\right)_{\mathrm{p}}$ varies with the equivalent wavelength $\lambda_{\mathrm{e}}$. In this study, the equivalent wavelength was estimated as $0.8 \AA$ from a slightly modified version of Tertian's method ${ }^{5}$, and regarded as a constant irrespective of the variety of samples.

Intensity of Compton scattering line and mass absorption coefficients

It is well known that the intensity of the Compton scattering line is linearly related to the reciprocal of the mass absorption coefficient. Results obtained with copper compounds and pure sulfur are shown in Fig. 2. The total intensity at the peak angle was taken as the Compton intensity, because of the large variation involved in background subtraction. The mass absorption coefficients $\left(\mu=\Sigma \mu_{\mathrm{i}} c_{\mathrm{i}}\right)$ for these specimens were computed from the following equation and the chemical compositions:

$$
\mu_{\mathrm{i}}=c_{\mathrm{i}} \lambda^{n \mathrm{i}}
$$

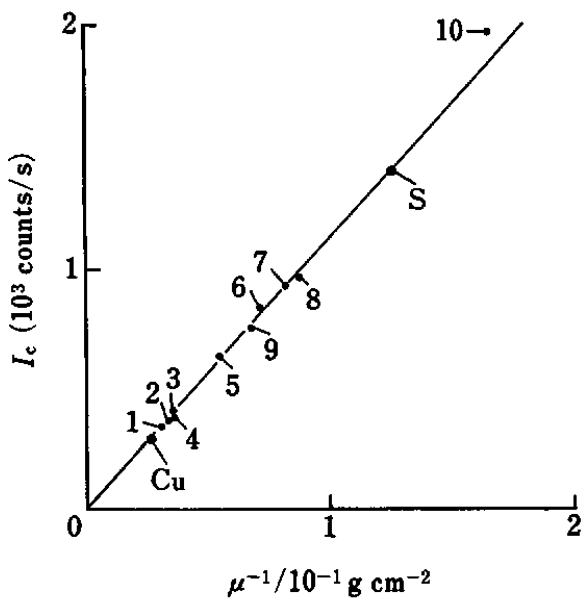

Fig. 2 Compton scattering intensity $I_{c}$ as a function of the reciprocal of mass absorption coefficient $\mu$. The numbers corresponds to the numbers in Table 2.

Table 3 Values of factors in Eq. (10)

\begin{tabular}{lc}
\hline Factor & Value \\
\hline$k_{\mathrm{s}}$ & 7.922 \\
$k_{\mathrm{e}}$ & 1.808 \\
$k_{\mathrm{f}}$ & 1.024 \\
$\left(\mu_{\mathrm{s}}\right)_{\mathrm{p}}-\left(\mu_{1}\right)_{\mathrm{p}}$ & 263.9 \\
$\left(\mu^{*}\right)_{\mathrm{p}}$ & 130.9 \\
$A$ & 1.476 \\
\hline
\end{tabular}

here $n_{\mathrm{i}}$ and $c_{\mathrm{i}}$ are both constant. i denotes the element contained in the sample. To calculate the mass absorption coefficient of hydrogen, the estimated values 0.34 and 1.13 were obtained for $c_{\mathrm{i}}$ and $n_{\mathrm{i}}$ respectively from the literature. ${ }^{6}$

\section{Calculation of factors}

The values $k_{\mathrm{s}}, k_{\mathrm{e}}, k_{\mathrm{f}},\left(\mu^{*}\right)_{\mathrm{p}}$ and $\left(\mu_{\mathrm{s}}\right)_{\mathrm{p}}-\left(\mu_{1}\right)_{\mathrm{p}}$ for copper were calculated using Eqs. (5), (7), (8) and (12). They are listed in Table 3.

\section{Results of quantitative analysis}

The results of quantitative analysis using Eq. (10) are shown in Table 4. For the analyte line, the $\mathrm{Cu} \mathrm{K} \mathrm{K}_{\beta}$ was used instead of $\mathrm{Cu} \mathrm{K}_{\alpha}$ in order to minimize the influence of detector dead time and to reduce errors arising from the application of Hower's relation by using a wavelength of the analyte line closest to the absorption edge. The maximum relative error was $+4.5 \%$ for copper(II) potassium chloride. The maximum absolute error was $-1.9 \%$ for copper(I) chloride.

\section{Source of errors}

The determination using Eq. (10) may involve the following sources of errors:

1) inaccuracy of mass absorption coefficients, especi- 
Table 4 Accuracy of absorption correction

\begin{tabular}{|c|c|c|c|c|c|c|}
\hline \multirow{2}{*}{$\begin{array}{c}\text { Sample } \\
\text { No. }\end{array}$} & \multirow{2}{*}{$R_{\mathrm{f}}$} & \multirow{2}{*}{$\begin{array}{c}\mu_{\mathrm{c}} \\
\mathrm{cm}^{2} \mathrm{~g}^{-1}\end{array}$} & \multicolumn{2}{|c|}{ Concentration, $\%$} & \multirow{2}{*}{$\begin{array}{c}\text { Error, } \\
\%\end{array}$} & \multirow{2}{*}{$\begin{array}{c}\text { Relative } \\
\text { error, } \\
\%\end{array}$} \\
\hline & & & Obsd. & Content & & \\
\hline 1 & 0.9801 & 33.99 & 88.5 & 88.8 & -0.8 & -0.9 \\
\hline 2 & 0.9735 & 30.98 & 80.9 & 79.9 & +1.0 & +1.3 \\
\hline 3 & 0.6757 & 27.66 & 65.4 & 66.5 & -1.1 & -1.7 \\
\hline 4 & 0.6242 & 28.98 & 66.8 & 64.2 & +2.6 & +4.0 \\
\hline 5 & 0.4674 & 17.70 & 36.8 & 37.3 & -0.5 & -1.3 \\
\hline 6 & 0.8740 & 13.46 & 34.2 & 35.3 & -1.1 & -3.1 \\
\hline 7 & 0.8717 & 12.14 & 30.8 & 31.8 & -1.0 & -3.1 \\
\hline 8 & 0.5974 & 11.56 & 26.3 & 25.5 & +0.8 & +3.1 \\
\hline 9 & 0.2071 & 14.96 & 20.8 & 19.9 & +0.9 & +4.5 \\
\hline 10 & 0.6978 & 5.68 & 13.6 & 14.0 & -0.4 & -2.9 \\
\hline
\end{tabular}

ally in a sample relatively thin compared to the critical depth;

2) calculation of mass absorption coefficients from Compton scattering;

3) application of Hower's relation;

4) estimation of equivalent wavelength;

5) inaccuracy of geometrical factors.

For 1), 2) and 3), it seems possible to reduce errors by using mass absorption coefficients of the same compilation for a sufficiently thick sample.

As a result of the examination in which the values of the equivalent wavelength and the geometrical factor were changed, it is found that the errors are insensitive to their values in the ranges $0.6<\lambda_{e}<1.0$ and $1.329<A<$ 2.214 .

\section{Limits to application of this method}

This analytical method can be applied only to the heaviest element in the light element matrix. When absorption edges of matrix elements lie near that of the analyte element, the mass absorption coefficients of fluorescent X-rays are large, and the estimation of equivalent wavelength becomes important. If the content of the heaviest element is small, the second term of the denominator of Eq. (10) is so small compared with the first term that it becomes negligible. In this case, this method is the same as Andermann's correction method. ${ }^{7}$ Thus the calibration curves can be estimated with the aid of mass absorption coefficient at Compton scattering wavelength.

We are pleased to acknowledge the support for this work given by the Asahi Glass Industrial Technology Promotion Fund.

\section{References}

1. R. C. Reynolds, Am. Mineral., 48, 1133 (1963).

2. K. K. Nielson, Anal. Chem., 49, 641 (1977).

3. T. Ui, M. Kataoka, M. Kato and E. Asada, Bunseki Kagaku, 37, 538 (1988).

4. J. Hower, Am. Mineral., 44, 19 (1959).

5. R. Tertian and F. Claisse, "Principles of Quantitative $X$-ray Fluorescence Analysis", pp. 260, 88 and 91, Heyden, London, 1982.

6. A. J. Bearden, Bull. Am. Phys. Soc., 4, Ser. II, 66 (1957).

7. G. Andermann and J. W. Kemp, Anal. Chem., 30, 1306 (1958).

(Received September 8, 1989)

(Accepted December 11, 1989) 